\title{
Thermal-hydraulic Analysis of Buffer System for Hydraulic Pile Hammers by Pseudo-bond Graph
}

\author{
Kejun $\mathrm{Li}^{1}$ and Junping $\mathrm{Hu}^{1}$ \\ ${ }^{1}$ College of Mechanical and Electrical Engineering, Central South University, \\ Changsha 410083, China \\ ntscss@163.com
}

\begin{abstract}
The damage of cylinder and equipment due to the pile detaching can be avoided by using the buffer device with large flow, which adopts throttle buffer inside the cylinder combining with the control of the relief valve outside the cylinder. To investigate the thermodynamic characteristics of the cylinder chambers and the law of movement of piston, the accurate mathematic model need to be established so the method based on pseudo-bond graph is introduced. According to the conservation equations for mass and energy, the thermalhydraulic pseudo bond graph $C$ and $R$ elements are developed, and the connection rule for the two elements are determined based on their input and output variables. The bond graph model of the buffer system containing the coupled of multiple energy domains is obtained in a unified way based on the structure and working principle of buffer device, then the system state equations have been derived from the model. The model can be used in variable parameters' simulation with the parameters in the experiment, the pressure simulation data of two chambers of impact cylinder agree well with the experimental results, which indicate that the model can be used for the design and optimization of buffer device of hydraulic pile hammer.
\end{abstract}

Keywords: hydraulic pile hammer, buffering device, pseudo bond graph, multi-energy coupling, simulation

\section{Introduction}

Hydraulic pile hammer is widely used in pile foundation construction for the advantages of low noise, low energy consumption and high efficiency. The impact cylinder with large flow is the key equipment of hydraulic pile hammer [1-2]. Once the hammer and pile separates in the working process, the piston will strike the cylinder cover with high speed and large inertia, which produces a great impact force to damage the cylinder and equipment. In order to avoid this phenomenon, a buffer device adopting throttle buffer inside the cylinder combining with the relief valve outside the cylinder is installed in the end of cylinder piston to enable the piston stop stably and safely. The impact energy is consumed and converted into heat by throttling in a very short time, so the temperature of hydraulic oil in the chamber rises sharply. To investigate the thermodynamics of the cylinder chamber and the law of movement of piston, it is necessary to establish a complete and accurate mathematical model for the buffer system.

Hydraulic hammer buffer system modeling involves multi-energy domains, such as hydraulic, mechanical and thermodynamic. The traditional modeling method [3-6], limited to a particular domain, failed to unify the physical quantity in different energy domain, so the application of the model is restricted to a certain extent. Consequently, other analytical 
approach such as bond graph method based on energy and information flow is considered to be more desirable in the present study. This allows one to study the structure of the system model. The nature of the parts of the model and the manner in which the parts interact can be made evident in a graphical format. The system equations also can be derived algorithmically in a systematic manner when the overall bond graph is ready [7-9]. Karnopp [10] proposed the concept of pseudo bond graph for the thermo-fluid systems, the temperature and pressure are defined as effort variables, the corresponding flow variables are energy flow and mass flow. Apart from the restriction that the product of the variables associated with each bond does not equal the instantaneous power, pseudo bond graphs share all other features with true bond graphs. But it is only applied on the study of compressible gas, the thermo-hydraulic system need to be further research.

In the present study, refer to the experience of pseudo bond graph used in the pneumatic system, the thermal-hydraulic pseudo bond graph $\mathrm{C}$ and $\mathrm{R}$ elements are developed based on the basic laws of physics, the mathematical principles and connection rule for the two elements are also studied. Subsequently, the bond graph model of pile hammer buffer system is constructed in a unified way, and the system state equations have been derived from this model. Finally, the mathematical model is verified experimentally, which provides a theoretical basis for the design and optimization for the buffer device of hydraulic pile hammer.

\section{Pseudo Bond Graph Elements for Thermal-hydraulic Model}

There is a wide variety of hydraulic components due to its function, working principle, structure, etc. However, consider the working mechanism of the hydraulic components [11], they can be divided into two types: the capacitive component and the resistance component.

\subsection{Pseudo Bond Graph C Element for the Capacitive Component}

The hydraulic pipeline, the cylinder chamber, the accumulator, etc, which are the basic capacitive component in hydraulic system. The basic capacitive component be regarded as the control volume in which the pressure and the temperature can be computed. The conservation equations for mass and energy are the basic equations for thermal-hydraulic modeling of the capacitive component. As shown in Figure 1, the changing rate of mass flow in the control volume can be described as

$$
\dot{m}=\sum \dot{m}_{i}-\sum \dot{m}_{o}
$$

Where, $\dot{m}_{i}$ is the flow rate of mass into the control volume, $\dot{m}_{o}$ is the rate of mass flow out of the control volume.

At any time, the mean fluid density in the control volume is given by

$$
\rho=m / V
$$

Combining Eq. (1), the time derivative of $\rho$ can be expressed as

$$
\dot{\rho}=\frac{\dot{m}-\rho \dot{V}}{V}
$$

Where, $V$ is the volume of the control volume.

Consider the thermodynamic properties of the fluid, the density can be expressed as a function of pressure and temperature

$$
\rho=\rho(P, T)
$$

By differentiating with respect to temperature and pressure, Eq. (4) leads to 

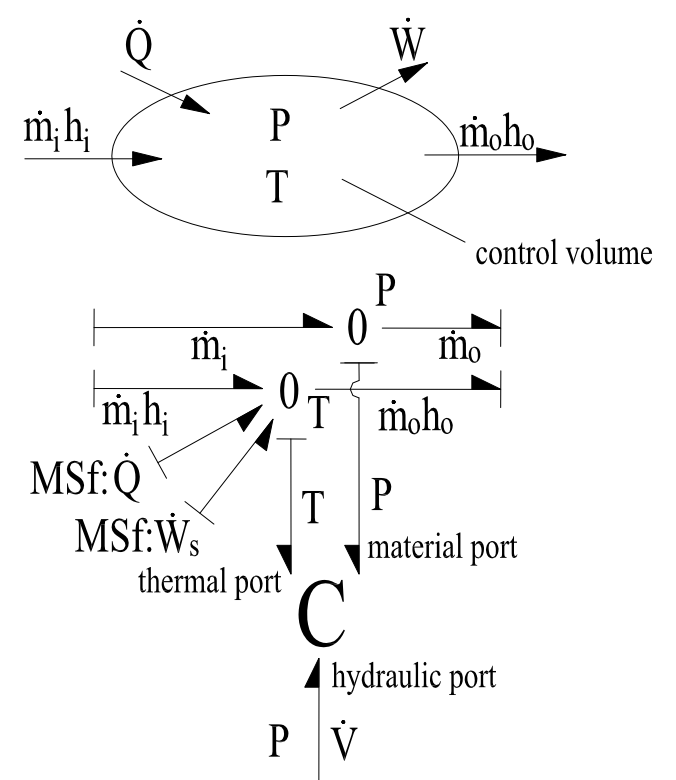

Figure 1. Pseudo Bond Graph C Element for the Control Volume

$$
d \rho=\left(\frac{\partial \rho}{\partial P}\right)_{T} d P+\left(\frac{\partial \rho}{\partial T}\right)_{P} d T
$$

From this equation, pressure in the control volume can be computed:

$$
d P=\frac{1}{\left(\frac{\partial \rho}{\partial P}\right)_{T}}\left[d \rho-\left(\frac{\partial \rho}{\partial T}\right)_{P} d T\right]
$$

The isothermal bulk modulus $\beta_{T}$ and the cubical expansion coefficient $\alpha_{p}$ are defined as follow

$$
\left\{\begin{array}{c}
\beta_{T}=\rho\left(\frac{\partial P}{\partial \rho}\right)_{T} \\
\alpha_{p}=-\frac{1}{\rho}\left(\frac{\partial \rho}{\partial T}\right)_{P}
\end{array}\right.
$$

Substituting Eq. (7) into Eq. (6) and differentiating with respect to time leads to

$$
\dot{P}=\beta_{T}\left[\frac{1}{\rho} \dot{\rho}+a_{P} \dot{T}\right]
$$

Combining Eq. (1), Eq. (3) and Eq. (8) gives

$$
\dot{P}=\beta_{T}\left[\frac{1}{\rho V}\left(\sum \dot{m}_{i}-\sum \dot{m}_{o}-\rho \dot{V}\right)+a_{P} \dot{T}\right]
$$

For one-dimensional flow, the energy conservation equation for the control volume can be written as:

$$
\dot{Q}-\dot{W}=\sum \dot{m}_{o} h_{o}-\sum \dot{m}_{i} h_{i}+\dot{E}
$$

Where $\dot{Q}$ represents heat flow absorbed by the control volume from outside, including heat transfer and heat radiation. $\dot{W}$ is the rate of work except for the work required to push mass 
into and out of the control volume, which is taken care of by using enthalpies for the energy of fluid streams. $\dot{E}$ is the time rate of change of the fluid energy in the control volume. $h_{i}$ is the specific enthalpy of the fluid flowing into the control volume. $h_{\mathrm{o}}$ is the specific enthalpy of the fluid flowing out of the control volume.

Energy in the control volume can also be expressed as:

$$
E=u+K E+P E
$$

Where, $u$ is the internal energy, $K E$ is the kinetic energy and $P E$ is the potential energy. Generally, $K E$ and $P E$ is very small compared with $u$, so they can be ignored. As a result, the time rate of change of the fluid energy in the control volume can rewritten as:

$$
\dot{E}=m \dot{u}+u \dot{m}
$$

It is assumed that fluid in the control volume do not change phase, so the fluid enthalpy can be defined as:

$$
h=u+P v
$$

Where, $u$ is the fluid enthalpy, $P$ is the fluid pressure, $v$ is the specific volume.

The time rate of change of the fluid enthalpy is calculated as follows [12]:

$$
\dot{h}=C_{P} \dot{T}+\left(1-\alpha_{P} T\right) v \dot{P}
$$

Where, $\dot{T}$ is the temperature of the fluid in the control volume, $\alpha_{P}$ is the fluid volume expansion coefficient, $C_{P}$ is the specific heat of the fluid.

After introducing Eq. (14) and Eq. (13) into Eq. (12), Eq. (15) is obtained:

$$
\dot{E}=c_{P} m \dot{T}-m T a_{P} v \dot{P}+h \dot{m}-P \dot{V}
$$

Combined Eq. (1), Eq. (10) and Eq. (15) gives

$$
\dot{T}=\frac{1}{c_{P} m}\left[\sum \dot{m}_{i}\left(h_{i}-h\right)+\sum \dot{m}_{o}\left(h-h_{o}\right)+\dot{Q}-\dot{W}+P \dot{V}+\frac{m T a_{p}}{\rho} \dot{P}\right]
$$

Where $\dot{W}$ represents the rate of boundary work and shaft work. It can be written as:

$$
\dot{W}=\dot{W}_{S}+\dot{W}_{b}=\dot{W}_{s}+P \dot{V}
$$

Introducing Eq. (16) into Eq. (15) gives:

$$
\dot{T}=\frac{1}{c_{P} m}\left[\sum \dot{m}_{i}\left(h_{i}-h\right)+\sum \dot{m}_{o}\left(h-h_{o}\right)+\dot{Q}-\dot{W}_{s}+\frac{m T a_{P}}{\rho} \dot{P}\right]
$$

Generally, it is assumed that the average enthalpy within the control volume equates to the leaving enthalpy regardless of the inlet conditions [13]. Eq. (18) can be rewritten as:

$$
\dot{T}=\frac{1}{c_{P} m}\left[\sum \dot{m}_{i}\left(h_{i}-h\right)+\dot{Q}-\dot{W}_{s}+T a_{P} V \dot{P}\right]
$$

Eq. (9) and Eq. (19) are the lumped parameter equations representing conservation of mass and energy.

Obviously, the two equations are cross coupled. Therefore, fast pressure transients will produce fast temperature transients and vice versa [14]. meanwhile, according to the two equations, state variables $\dot{P}$ and $\dot{T}$ are defined as effort variables of the pseudo bond graph C element, and state variables $\dot{m}, \dot{V}, \dot{Q}$ and $\dot{W}_{s}$ can be defined as flow variables of the pseudo bond graph $\mathrm{C}$ element (as show in Figure 1 below). The $\mathrm{C}$ element has three ports, named thermal port, material port and hydraulic port respectively. Summation of flows at the 0junction connected to the thermal port of the C element yields the energy balance of Eq. (19), the second 0-junction connected to the material port represents the mass balance of Eq. (9). The thermodynamic accumulator can be linked with a bond graph of a mechanical subsystem via a transformer [15]. 


\subsection{Pseudo Bond Graph R Element for the Resistance Component}

As shown in Figure 2, the throttle valve, directional valve, oil filter, etc, which are typical resistance component. The mass flow rate through the resistive components depends on the concrete resistive structure and the fluid state. For example, the standard orifice turbulent mass flow equation is given by [17]

$$
\dot{m}=\rho \dot{V}=\rho c_{d} A \sqrt{2 \rho\left(P_{1}-P_{2}\right)}
$$

It is assumed that the fluid enthalpy of the flow through the resistive component do not change, so the power consumption converted into heat can be computed by

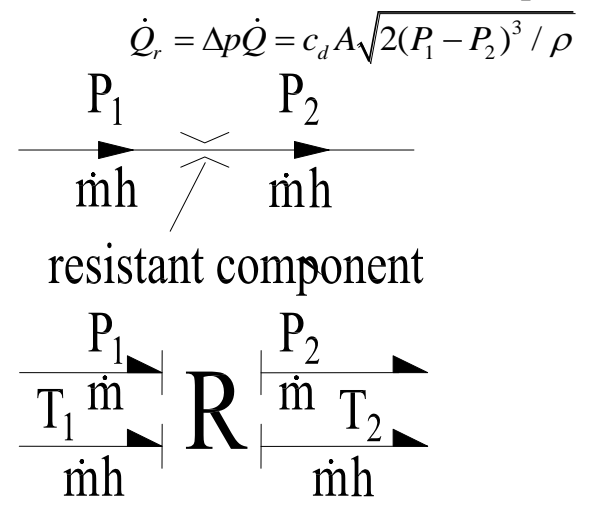

Figure 2. Pseudo Bond Graph R Element for the Resistance Component

According to the above two equations, state variables $\dot{P}$ and $\dot{T}$ are defined as effort variables of the pseudo bond graph $\mathrm{R}$ element, the state variables $\dot{m}, \dot{V}, \dot{Q}$ and $\dot{W}_{s}$ can be defined as flow variables of the pseudo bond graph $\mathrm{R}$ element. It is apparent that the input variables of resistive component are pressure and temperature, which are just the output variables of capacitive component, the output variable of resistive component are mass flow rate and heat flow rate, which can be used in the capacitive component, so the connection rule for the two pseudo bond graph elements can be determined as shown in Figure 3.

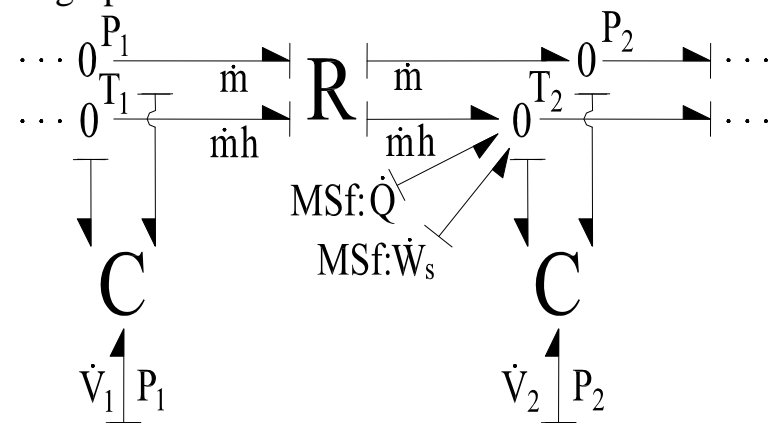

Figure 3. Connection Rule for the Two Pseudo Bond Graph Elements

\subsection{Heat Transfer Calculation}

In hydraulic system, the control volume is a closed space surrounded by metal shell or hydraulic pipelines. If the temperature difference exists between the fluid in the control volume and the shell, the heat transfer will occur inevitably by convection. The heat exchange between the control volume and the shell is given as

$$
\dot{Q}_{t}=K A\left(T_{f}-T_{w}\right)
$$


Where, $K$ is the fluid/shell heat transfer coefficient, $A$ is the heat transfer area inside the shell, $T_{f}$ is the fluid temperature, and $T_{w}$ is the shell temperature. In Eq. (21) and Eq. (22), it can be seen that the heat transferred to the control volume consists of two parts, one part is generated by the resistant components, the other part is transferred from the body shell.

\section{Description of Buffer System}

\subsection{The Structure of Buffer Device}

As shown in the Figure 4, the buffer device with large flow is installed at the end of the impact cylinder stroke, which is composed of cylinder cover, guide sleeve, spring, stopper, floating piston, check valve and so on. The buffer chamber pressure area should satisfy the requirement for the damping force produced by the buffer device. One end of spring is fixed on an annular of guide sleeve, and the other end is pressed on the stopper. Two check valves are designed on the floating piston. The pre-compression force of spring keeps the floating piston in contact with cylinder jacket. Buffer chamber is connected with rod chamber by two check valves and the relief valve, the hydraulic oil in the rod chamber can flow into the buffer chamber through check valve, and the fluid in the buffer chamber can flow into the rod chamber through relief valve and oil joint.

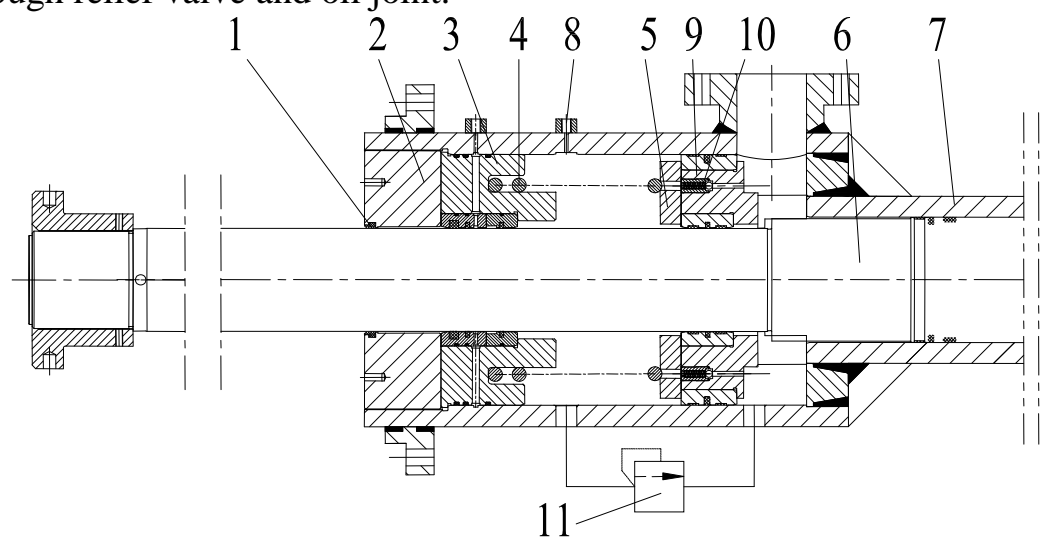

1.dust seal 2.cylinder cover 3.guide sleeve 4.spring 5.stopper 6.piston 7.cylinder jacket 8.oil joint 9.floating piston 10.check valve 11.relief valve

Figure 4. Structure Schema for the Buffer Device

\subsection{The Working Principle of Buffer System}

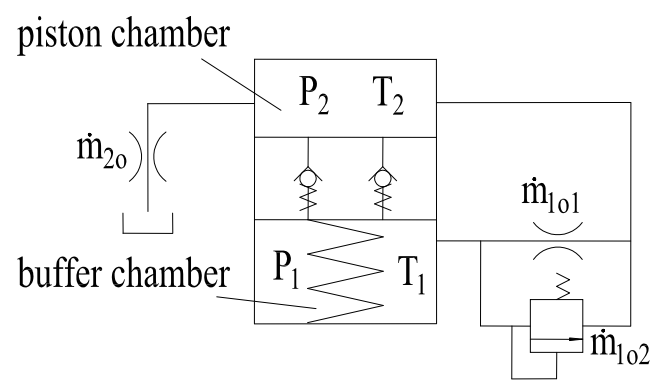

Figure 5. The Working Principle of the Buffer Device 
The working principle of buffer system is shown in Figure 5. When the piston of impact cylinder collides with the floating piston, they move downward together. The fluid in the buffer chamber is compressed by the floating piston. With the pressure increasing in the buffer chamber, the fluid flows through the oil joint and into the rod chamber. The damping effect of the return pipe can considered as a resistance component. When the pressure in the buffer chamber reaches the setting pressure of the relief valve, the relief valve will open to protect the buffer chamber. Finally, the impact energy will be consumed and converted into heat by the throttling effect of the oil joint and the relief valve.

\section{Hammers Buffer System Modeling}

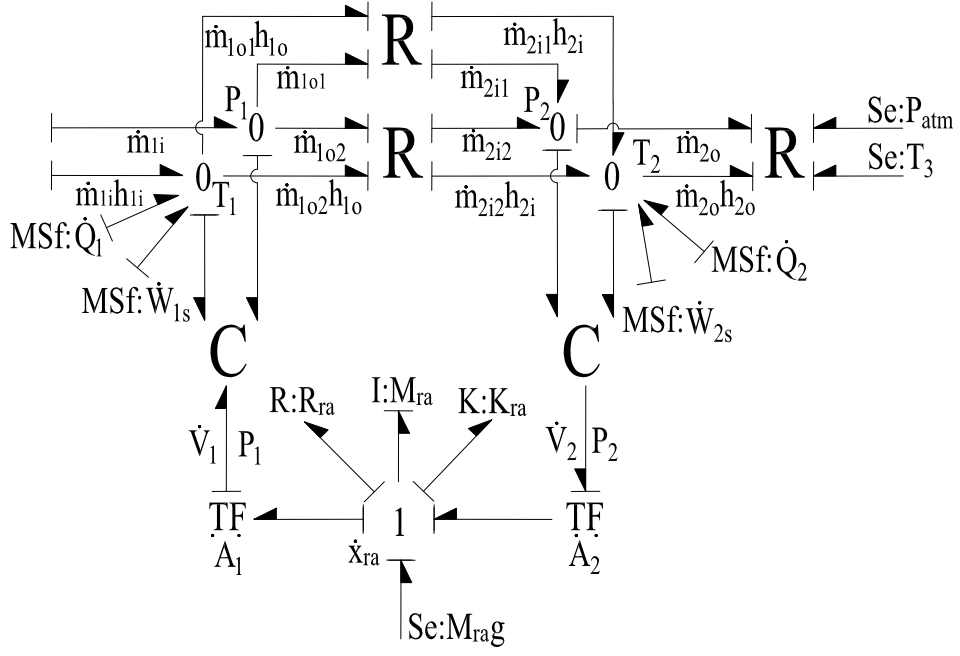

Figure 6. The Bond Graph Model of Buffer System

According to the bond graph theory, the various components of the buffer system can be abstracted to be bond graph elements, then the bond graph model of buffer system have been developed as shown in Figure 6. In this model, hydraulic system and mechanical system are linked by the two pseudo bond graph $\mathrm{C}$ element via the TF element. The transformer moduli $A_{1}, A_{2}$ are the effective cross-sectional area on both sides of the floating piston. The $1\left(_{\dot{x}_{r a}}\right.$ ) junction represents the speed of the piston, $K_{r a}$ and $R_{r a}$ represent the stiffness of the spring and the damping coefficient of the piston. $M_{r a}$ is the equivalent mass of the piston and pile hammer. The effort source $S_{e}\left(M_{r a} g\right)$ represents the gravity of the piston. $P_{1}$ and $T_{1}$ are the pressure and temperature of fluid in the buffer chamber, respectively. $P_{2}$ and $T_{2}$ are the pressure and temperature of fluid in the piston chamber, respectively. The $R_{o r}$ element indicates the resistance of throttle valve, the flow $\dot{V}_{o r}$ through which depends on the port opening area. The $R_{r f}$ element represents the resistance of relief valve, through which the flow $\dot{V}_{r f}$ passes to piston chamber. The hydraulic resistance of return pipeline is taken into account by $R_{r e}$ element, through which the flow $\dot{V}_{r e}$ passes to piston chamber. Specially, the buffer action is completed in a very short time, so the heat transfer between the control volume and the wall can be ignored. The system equations derived from the model are as follows:

The force equilibrium equation of the floating piston is given as follows: 


$$
\dot{P}_{r a}=P_{2} A_{2}-P_{1} A_{1}+M_{r a} g-K_{r a}\left(x_{r a}+x_{p r a}\right)-R_{r a} \dot{x}_{r a}
$$

Where, $x_{r a}$ and $x_{p r a}$ are the displacement of floating piston and the pre-compression of the spring, respectively.

The velocity of the floating piston is expressed as:

$$
\dot{x}_{r a}=P_{r a} / M_{r a}
$$

The time derivative of the pressure and temperature of fluid in the buffer chamber can be expressed as:

$$
\begin{aligned}
& \dot{P}_{1}=\beta_{T}\left[\frac{1}{\rho V_{1}}\left(\dot{m}_{1 i}-\dot{m}_{1 o 1}-\dot{m}_{1 o 2}-\rho \dot{V}_{1}\right)+a_{p} \dot{T}_{1}\right] \\
& \dot{T}_{1}=\frac{1}{c_{p} m_{1}}\left[\dot{m}_{1 i}\left(h_{1 i}-h_{1}\right)+\dot{Q}_{1}-\dot{W}_{1 s}+\dot{V}_{1} T_{1} a_{p} \dot{P}_{1}\right]
\end{aligned}
$$

Where, $V_{1}$ is the volume of the buffer chamber. $m_{1}$ is the mass of fluid in the buffer chamber. $\dot{m}_{101}$ and $\dot{m}_{102}$ is the mass flow through the throttle valve and the relief valve, respectively. $\dot{m}_{101}$ is the mass flow passes into the buffer chamber. $h_{1 i}$ is the specific enthalpy of the fluid flowing into the buffer volume, $h_{1}$ is the specific enthalpy of the fluid in the buffer volume. $\dot{Q}_{1}$ represents the heat change between the buffer chamber and the cylinder wall and equals zero. $\dot{W}_{1 s}$ is the shaft power of the buffer chamber.

The volume of piston chamber is computed by

$$
V_{1}=\frac{\pi}{4}\left(D^{2}-d^{2}\right)\left(L-x_{r d}\right)
$$

Where, $D$ is the inner diameter of the piston chamber. $d$ is the diameter of the piston rod. $L$ is the length of the buffer chamber.

The mass flow through the throttle hole can be expressed as:

$$
\dot{m}_{101}=\rho C_{j} \frac{\pi}{4} d_{j}^{2} \sqrt{2\left(P_{1}-P_{2}\right) / \rho}
$$

Where, $C_{j}$ is the flow coefficient through throttle hole. $d_{j}$ is the diameter of the throttle hole.

The mass flow through the relief valve can be expressed as:

$$
\dot{m}_{102}=\left\{\begin{array}{cl}
0 & P<P_{s e t} \\
\rho C_{y} \frac{\pi}{4} A_{y} \sqrt{2\left(P_{1}-P_{2}\right) / \rho} & P \geq P_{s e t}
\end{array}\right.
$$

Where, $C_{y}$ is the flow coefficient through the relief valve. $A_{y}$ is the area of the relief valve.

The time derivative of the pressure and temperature in the piston chamber can be expressed as

$$
\begin{gathered}
\dot{p}_{2}=\beta_{T}\left[\frac{1}{\rho V_{2}}\left(\dot{m}_{2 i 1}+\dot{m}_{2 i 2}-\dot{m}_{2 o}-\rho \dot{V}_{2}\right)+a_{p} \dot{T}_{2}\right] \\
\dot{T}_{2}=\frac{1}{c_{p} m_{2}}\left[\left(\dot{m}_{2 i 1}+\dot{m}_{2 i 2}\right)\left(h_{2 i}-h_{2}\right)+\dot{Q}_{2}-\dot{W}_{2 s}+\dot{V}_{2} T_{2} a_{p} \dot{P}_{2}\right]
\end{gathered}
$$

Where, $V_{2}$ is the volume of the piston chamber. $m_{2}$ is the mass of fluid in the piston chamber. $\dot{m}_{2 o}$ is the mass flow through the resistance $R_{r e} . \dot{m}_{2 i 1}$ and $\dot{m}_{2 i 2}$ is the mass flow through the throttle hole and the relief valve, and $\dot{m}_{2 i 1}$ is equal to $\dot{m}_{101}, \dot{m}_{2 i 2}$ is equal to $\dot{m}_{102} . h_{2 i}$ is the 
specific enthalpy of the fluid flowing into the piston volume, $h_{2}$ is the specific enthalpy of the fluid in the piston volume. $\dot{Q}_{2}$ is the heat transfer from buffer chamber to piston chamber. $\dot{W}_{2 s}$ is the shaft power of the piston chamber.

The volume of piston chamber is given by

$$
V_{2}=V_{20}+\frac{\pi}{4}\left(D^{2}-d^{2}\right) x_{r d}
$$

Where $V_{20}$ is the initial volume of the piston chamber.

The mass flow through the return pipe can be computed by

$$
\dot{m}_{2 o}=\rho\left(P_{2}-P_{a t m}\right) / R_{r e}
$$

The heat entering the piston chamber is expressed as

$$
\dot{Q}_{2}=C_{j} \frac{\pi}{4} d_{j}^{2} \sqrt{2\left(P_{1}-P_{2}\right)^{3} / \rho}+C_{y} \frac{\pi}{4} A_{y} \sqrt{\left.2\left(P_{1}-P_{2}\right)^{3} / \rho\right)}
$$

\section{Simulation Results and Discussions}

The simulation on the dynamic performance of the buffer system has been carried out by solving the system equations obtained in Section 4 numerically. Two pressure sensors are

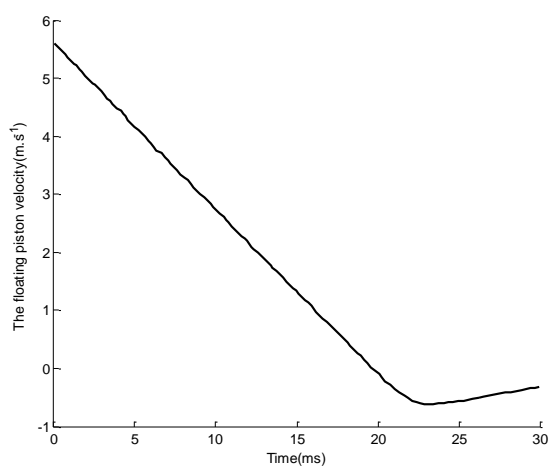

(a) The floating piston velocity

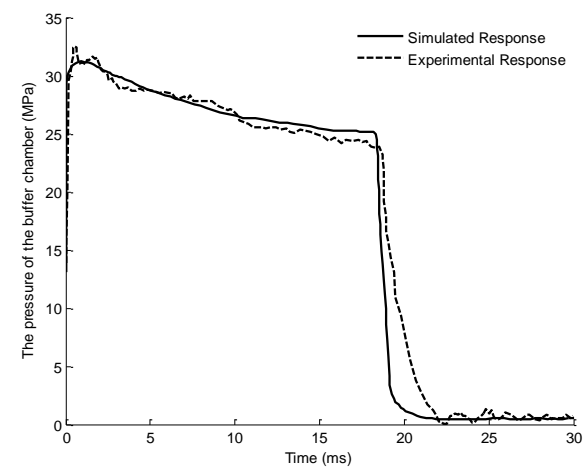

(c) The pressure in the buffer chamber

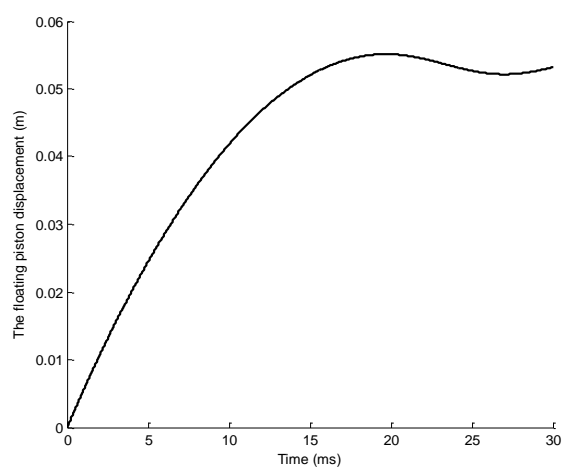

(b) The floating piston displacement

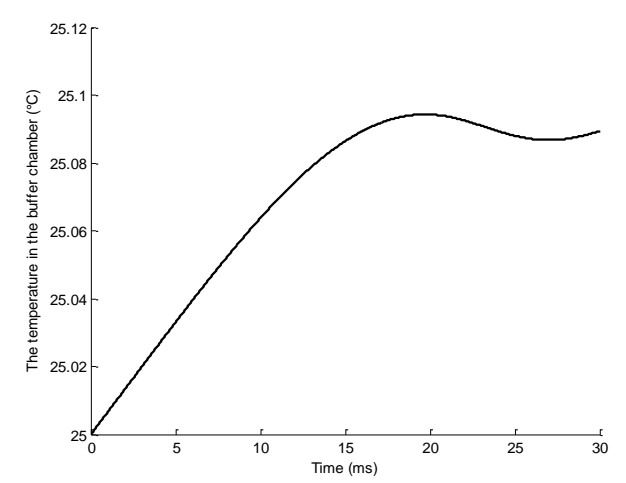

(d) The temperature in the buffer chamber 


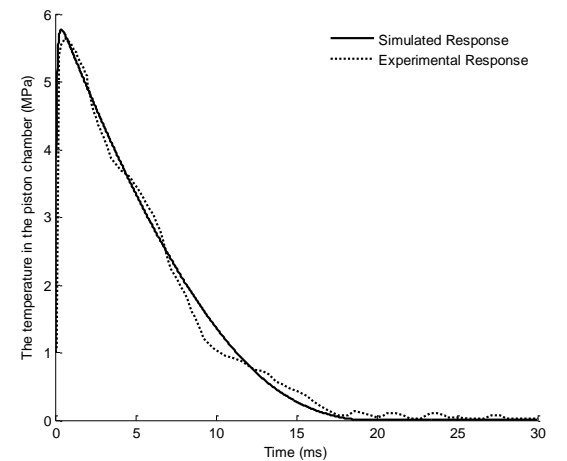

(e) The pressure in the piston chamber

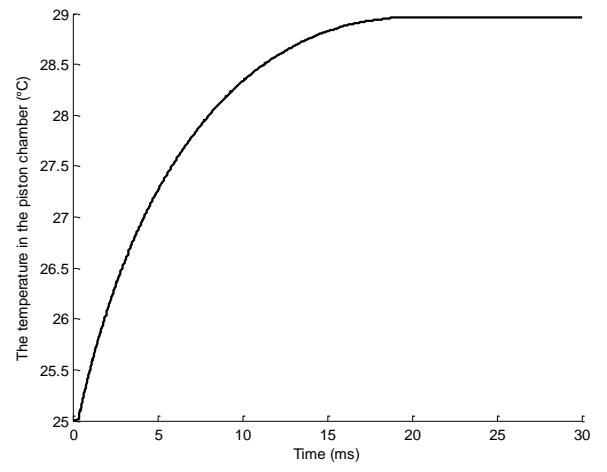

(f) The temperature in the piston chamber

Figure 7. The Dynamic Response of Buffer System

installed at the outlet port of the impact cylinder to test the validity of the modeling method. The two chambers pressure value is recorded by the data acquisition card, then, the pressure curves are drawn with the help of computer. The total simulation time is $30 \mathrm{~ms}$. The simulation results are shown in Figure 8. The following conclusions can be obtained by analysis

(1) Figure 8(a) shows the floating piston velocity response. It can be seen that the velocity of the floating piston is zero at about $19.8 \mathrm{~ms}$, which is much less than the duty cycle of pile driving work(1 2s), meanwhile, the buffer system have finished the absorption of the impact energy. Therefore, the buffer device meets the time requirement of design.

(2) Figure 8(b) shows the floating piston displacement response. In the buffering process, the maximum displacement of the floating piston is about $5.55 \times 10^{-2} \mathrm{~m}$, which is $2.05 \times 10^{-2} \mathrm{~m}$ shorter than the design value, this finding indicates that the buffer device satisfies the requirement of the size of the structural design.

(3) Figure 8(c) and (e) show the pressure response in the two chambers of impact cylinder. At $1.3 \mathrm{~ms}$, the pressure in the buffer chamber reaches about $31 \mathrm{MPa}$, this means that the maximum acceleration in the floating piston is about 32 times the acceleration of gravity, and the pressure of the piston

chamber is only about $5.7 \mathrm{Mpa}$. The results confirm that the number of throttling holes or the opening area of the relief valve should be increased to reduce the peak pressure of buffer chamber.

(4) Comparing the experimental and simulation pressure results of the two chambers, it can be found that there are about $10 \%$ errors between the simulated and tested pressure curves. This is due to the random noise interfere during the pressure data acquisition, what's more, it's difficult to give exact values of some parameters, such as the movement damping coefficient of the cylinder piston, valve port flow coefficient, the opening time of the relief valve, etc. However, the agreement between the simulated and tested results is acceptable for the application in this paper, this means that the model can be used to analyze and predict the dynamic characteristics of the system.

(5) According to the temperature response of the two chambers as shown in Fig 8. (d) and (f), it can be seen that the maximum temperature of the piston chamber reaches $28.9^{\circ} \mathrm{C}$, which is $3.9^{\circ} \mathrm{C}$ higher than the initial temperature, this depends on a large amount of heat generated by the throttle hole and relief valve, while, the temperature in the buffer chamber was 
$25.09^{\circ} \mathrm{C}$, only about $0.09^{\circ} \mathrm{C}$ higher than the initial temperature. In the buffering process, the less work acted by the gravity is the main reason for this difference. That's why the impact energy of the impact cylinder is mainly absorbed by the throttling effect of the relief valve and the throttle hole.

From the above analysis, it can be concluded that the model can fully reveal the energy input, transfer, translation and dissipation of the buffer system. Meanwhile, the model can be used to analyze and optimize the buffer device by modifying the system parameters, which will be studied in the future research.

\section{Conclusion}

(1) Firstly, according to the working mechanism of hydraulic components, they can be separated into two categories: capacitive components and resistive components, then the thermal-hydraulic pseudo bond graph $\mathrm{C}$ and $\mathrm{R}$ elements for the capacitive connection rule for basic thermal-hydraulic pseudo bond graph elements and the method to automatically generate the complete thermal-hydraulic system model are put forward, which are applied to the multi-energy model of buffer system of hydraulic pile hammer. The model is verified experimentally, which can fully reveal the working performance and dynamic characteristics of the buffer system and provide a theoretical basis for the optimization design of buffer device.

(2) The pseudo bond graph $\mathrm{C}$ and $\mathrm{R}$ elements are basic units for the thermodynamic analysis of hydraulic system. The hydraulic component can be abstracted as capacitive elements and resistant elements according to the working principle of the hydraulic circuit, then the complete bond graph model of hydraulic system can be obtained quickly by the connection rules. This modeling method provides a new research idea for the temperature simulation of hydraulic system and expands the simulation range for the bond graph theory. In addition, by using hydraulic port of pseudo bond graph $\mathrm{C}$ element, it is easy to joint with the subsystems of electromechanical part to construct a global coupled dynamic model of the system.

\section{Acknowledgements}

This work was supported by the National Natural Science Foundation of China (NO. 51175518).

This paper is a revised and expanded version of a paper entitled "A Novel Bond Graph Based Model for Buffer System" presented at The 3rd International Conference on Information Science and Industrial Applications (ISI 2014), held on August 22-24, 2014 at Shangda Hotel, Harbin, China.

\section{References}

[1] Y. Guo, J. P. Hu and L. Y. Zhang, "Finite-element analysis of multi-body contacts for pile driving using a hydraulic pile hammer", Proceeding of the Institution of Mechanical Engineers, Part C: Journal of Mechanical Engineering Science, vol. 255, no. 5, (2011), pp. 1153-1161.

[2] J. P. Hu, Y. Guo, G and W. Song, "Numerical Analysis for Impact Model of Pile Hammer Filled with Metal Particles", China Journal of Highway and Transport, vol. 23, no. 2, (2010), pp. 122-126.

[3] Z. Zhang, R. X. Zhou and Z. J. Wang, "Landing Gear Retraction System of Modular Modeling and Thermalhydraulic Simulation”, Modular Machine Tool \& Automatic Manufacturing Technique, vol. 3, (2013), pp. 36-38. 
[4] W. L. Wang and G. X. Xu, "Fluid formulae for damping changeability conceptual design of railway semiactive hydraulic dampers", International Journal of Non-Linear Mechanics, vol. 44, no. 7, (2009), pp.809819.

[5] J. N. Yao, W. Fu and S. H. Hu, "Amplitude phase control for electro-hydraulic servo system based on normalized least-mean-square adaptive filtering algorithm”, J. Cent. South Univ. Technol, vol. 18, (2011), pp.755-759.

[6] M. H. Han, K. Q. Cao and L. M. Hu, "Thermal Model and Simulation on Hydraulic Piston Pump Based on AMESim”, MACHINE TOOL \& HYDRAULIC, vol. 40, no. 1, (2012), pp.136-138.

[7] A. K. Samantaray and B. O. Bouamama, Model-based Process Supervision-A Bond Graph Approach, Springer Verlag, London, (2008), pp.22-43.

[8] M. Mandal and S. K. Pal, "Pseudo-bond graph modeling of temperature distribution in a through-process steel rolling", Mathematics and Computers in Simulation, 2008, vol. 77, no. 1, (2008), pp. 81-95.

[9] W. S. Ding and X. D. Huang, "Simulation Modeling of Nitrogen-Inflating Hydraulic Hammer", Journal of South China University of Technology, vol. 38, no. 2, (2010), pp.90-94.

[10] D. C . Karnopp, D. L. Margolis and R. C. Rosenberg, System Dynamics: Modeling, Simulation, and Control of Mechatronic Systems, John Wiley \& Sons, New York, (2012), pp.548-590.

[11] Y. L. Li, K. Q. Cao, H. J. Xu and X. B. Su, "Thermal-hydraulic Modeling and Simulation of Hydraulic System Based on Dymola”, Journal of System Simulation, vol. 2, no. 9, (2010), pp.2043-2047.

[12] W. Van and R. E. Sonntag, Fundamentals of classical thermodynamics, John Wiley \& Sons, New York, (1985).

[13] J. A. Sidders, D. G. Tilley and P. Chappie, "Thermal-hydraulic performance prediction in fluid power systems", Proceedings of the Institution of Mechanical Engineers, Part I: Journal of Systems and Control Engineering, vol. 210, no. 4, (1996), pp.231-242.

[14] C. G. Li and Z. X. Jiao, "Calculation method for thermal-hydraulic system simulation", Journal of Heat Transfer, vol. 130, no. 8, (2008), pp. 084503.

[15] W. Borutzky, "Bond graph methodology: development and analysis of multidisciplinary dynamic system models" Springer Verlag, London, (2009).

[16] Shakeel Ahmad, Bashir Ahmad, Sheikh Muhammad Saqib and Rashid Muhammad Khattak. Trust Model: Cloud's Provider and Cloud's User. International Journal of Advanced Science and Technology. 44 (2012).

[17] J. Watton, "Fundamentals of Fluid Power Control", CAMBRIDGE UNIVERSITY PRESS, (2009).

[18] Q. L. Jia, D. L. Yun and Y. F. Luan, "System simulation and design based on MATLAB7.x $\mid$ Simulink $\backslash$ Stateflow, Northwest Industrial University Press, (2006).

[19] P. X. Jiang, M. H. Fan and M. H. Si, "Thermal-hydraulic performance of small scale mico-channel and porous media heat exchanges", International Journal of Heat and Mass Transfer, vol. 44, no. 5, (2001), pp.1039-1051.

[20] A. K. Samantaray, "Modelling and analysis of preloaded liquid spring damper shock absorbers", Simulation Modelling Practice and Theory, 2009, vol. 17, no. 1, (2009), pp.309-325.

\section{Authors}

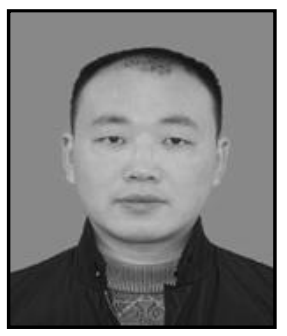

Kejun Li, he is a $\mathrm{PhD}$ Candidate of College of Mechanical and Electrical Engineering in Central South University. His research interests include fluid power components and mechatronic system design and hydraulics, in particular piling machinery hydraulic control systems.

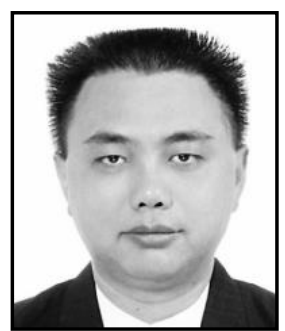

Junping Hu, he is a full professor of Mechanical and Electrical Engineering in Central South University. His present research interests include hydraulic transmission, energy saving and regeneration, and motion control. 